\title{
NTRK1 Fusion Positive
}

National Cancer Institute

\section{Source}

National Cancer Institute. NTRK1 Fusion Positive. NCI Thesaurus. Code C131070.

Indicates that the expression of a NTRK1 fusion has been detected in a sample. 